See discussions, stats, and author profiles for this publication at: https://www.researchgate.net/publication/321133323

\title{
Advanced Virgo Status
}

Conference Paper · December 2017

DOI: 10.1142/9789813226609_0406

\section{CITATIONS}

0

241 authors, including:

Teneisha Adams

Columbia College SC

411 PUBLICATIONS 24,981 CITATIONS

SEE PROFILE

P. Astone

INFN - Istituto Nazionale di Fisica Nucleare

474 PUBLICATIONS 20,539 CITATIONS

SEE PROFILE
READS

77

Some of the authors of this publication are also working on these related projects:

Regular Black Holes View project

Project Virgo/LSC View project

Annalisa Allocca

Università degli Studi di Siena

159 PUBLICATIONS 9,950 CITATIONS

SEE PROFILE

Philippe Bacon

Paris Diderot University

157 PUBLICATIONS 13,349 CITATIONS

SEE PROFILE 


\section{Advanced Virgo Status}

F. Acernese ${ }^{1,2}$, T. Adams ${ }^{3}$, M. Agathos ${ }^{4}$, K. Agatsuma ${ }^{4}$, L. Aiello ${ }^{5,6}$, A. Allocca $^{7,8}$, N. Arnaud ${ }^{9}$, S. Ascenzi ${ }^{10,6}$, P. Astone ${ }^{11}$, P. Bacon ${ }^{12}$, M. K. M. Bader ${ }^{4}$, F. Baldaccini ${ }^{13,14}$, G. Ballardin ${ }^{15}$, F. Barone ${ }^{1,2}$, M. Barsuglia ${ }^{12}$, D. Barta ${ }^{16}$, A. Basti ${ }^{7,8}$, V. Bavigadda ${ }^{15}$, M. Bazzan ${ }^{17,18}$, M. Bejger ${ }^{19}$, D. Bersanetti ${ }^{20,21}$, A. Bertolini ${ }^{4}$, M. Bitossi ${ }^{15}$, M. A. Bizouard ${ }^{9}$, S. Bloemen ${ }^{22}$, M. Boer ${ }^{23}$, G. Bogaert ${ }^{23}$, F. Bondu ${ }^{24}$, R. Bonnand ${ }^{3}$, B. A. Boom ${ }^{4}$, V. Boschi ${ }^{7,8}$, Y. Bouffanais ${ }^{12}$, A. Bozzi ${ }^{15}$, C. Bradaschia ${ }^{8}$, M. Branchesi ${ }^{25,26}$, T. Briant ${ }^{27}$, A. Brillet ${ }^{23}$, V. Brisson ${ }^{9}$, T. Bulik ${ }^{28}$, H. J. Bulten ${ }^{29,4}$, D. Buskulic ${ }^{3}$, C. Buy ${ }^{12}$, G. Cagnoli ${ }^{30,31}$,

E. Calloni ${ }^{32,2}$, E. Capocasa ${ }^{12}$, F. Carbognani ${ }^{15}$, J. Casanueva Diaz ${ }^{9}$, C. Casentini ${ }^{10,6}$, F. Cavalier ${ }^{9}$, R. Cavalieri ${ }^{15}$, G. Cella ${ }^{8}$, L. Cerboni Baiardi ${ }^{25,26}$, G. Cerretani ${ }^{7,8}$, E. Cesarini ${ }^{10,6}$, E. Chassande-Mottin ${ }^{12}$, A. Chincarini ${ }^{21}$, A. Chiummo ${ }^{15}$, S. Chua ${ }^{27}$, F. Cleva ${ }^{23}$, E. Coccia ${ }^{10,5,6}$, P.-F. Cohadon ${ }^{27}$, A. Colla ${ }^{33,11}$, A. Conte ${ }^{33,11}$, L. Conti ${ }^{18}$, S. Cortese ${ }^{15}$, J.-P. Coulon ${ }^{23}$, E. Cuoco $^{15}$, S. D'Antonio ${ }^{6}$, V. Dattilo ${ }^{15}$, M. Davier ${ }^{9}$, R. Day ${ }^{15}$, G. Debreczeni ${ }^{16}$, J. Degallaix ${ }^{30}$, M. De Laurentis ${ }^{32,2}$, S. Deléglise ${ }^{27}$, R. De Rosa ${ }^{32,2}$, L. Di Fiore ${ }^{2}$, M. Di Giovanni ${ }^{34,35}$,

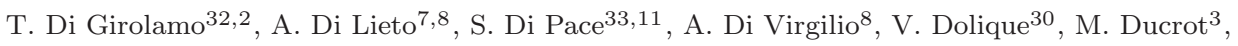
V. Fafone ${ }^{10,6,5, *}$, S. Farinon ${ }^{21}$, I. Ferrante ${ }^{7,8}$, F. Ferrini ${ }^{15}$, F. Fidecaro ${ }^{7,8}$, I. Fiori ${ }^{15}$, D. Fiorucci ${ }^{12}$, R. Flaminio ${ }^{30,36}$, J.-D. Fournier ${ }^{23}$, S. Frasca ${ }^{33,11}$, F. Frasconi ${ }^{8}$, V. Frey ${ }^{9}$,

L. Gammaitoni ${ }^{13}$, F. Garufi ${ }^{32,2}$, G. Gemme ${ }^{21}$, E. Genin ${ }^{15}$, A. Gennai ${ }^{8}$, V. Germain ${ }^{3}$, S. Ghosh ${ }^{22,4}$, A. Giazotto ${ }^{8}$, J. M. Gonzalez Castro ${ }^{7,8}$, M. Gosselin ${ }^{15}$, R. Gouaty ${ }^{3}$, A. Grado ${ }^{37,2}$,

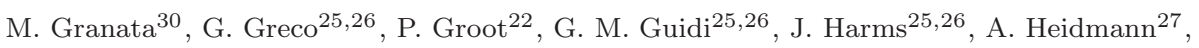

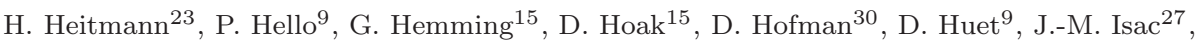
T. Jacqmin ${ }^{27}$, P. Jaranowski ${ }^{38}$, R. J. G. Jonker ${ }^{4}$, M. Kasprzack ${ }^{15}$, F. Kéfélian ${ }^{23}$, I. Khan ${ }^{5}$, S. Koley ${ }^{4}$, I. Kowalska ${ }^{28}$, A. Królak ${ }^{39,40}$, A. Kutynia ${ }^{39}$, C. Lazzaro ${ }^{18}$, P. Leaci ${ }^{33,11}$, E. O. Lebigot ${ }^{12}$, M. Leonardi ${ }^{34,35}$, N. Leroy ${ }^{9}$, N. Letendre ${ }^{3}$, M. Lorenzini ${ }^{5,6}$, V. Loriette $^{41}$, G. $\operatorname{Losurdo}^{26}$, E. Majorana ${ }^{11}$, I. Maksimovic ${ }^{41}$, V. Malvezzi ${ }^{10,6}$, N. Man $^{23}$, M. Mantovani $^{15}$, F. Marchesoni ${ }^{42,14}$, F. Marion ${ }^{3}$, F. Martelli ${ }^{25,26}$, L. Martellini ${ }^{23}$, A. Masserot ${ }^{3}$,

S. Mastrogiovanni ${ }^{33,11}$, D. Meacher ${ }^{23}$, J. Meidam ${ }^{4}$, M. Merzougui $^{23}$, R. Metzdorff ${ }^{27}$, F. Mezzani ${ }^{11,33}$, C. Michel ${ }^{30}$, L. Milano ${ }^{32,2}$, A. Miller ${ }^{33,11}$, Y. Minenkov ${ }^{6}$, A. Moggi ${ }^{8}$, M. Mohan ${ }^{15}$, M. Montani ${ }^{25,26}$, B. Mours ${ }^{3}$, I. Nardecchia ${ }^{10,6}$, L. Naticchioni ${ }^{33,11}$, G. Nelemans ${ }^{22,4}$, M. Neri ${ }^{20,21}$, S. Nissanke ${ }^{22,4}$, F. Nocera $^{15}$, C. Palomba ${ }^{11}$, F. Paoletti ${ }^{15,8}$, A. Paoli ${ }^{15}$, A. Pasqualetti ${ }^{15}$, R. Passaquieti ${ }^{7,8}$, D. Passuello ${ }^{8}$, B. Patricelli ${ }^{7,8}$, R. Pedurand ${ }^{30,43}$,

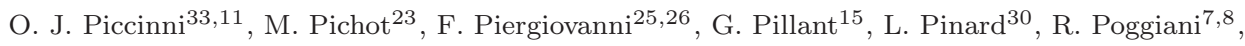
P. Popolizio ${ }^{15}$, M. Prijatelj ${ }^{15}$, G. A. Prodi ${ }^{34,35}$, M. Punturo ${ }^{14}$, P. Puppo ${ }^{11}$, P. Rapagnani ${ }^{33,11}$,

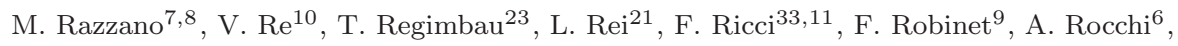

L. Rolland ${ }^{3}$, R. Romano ${ }^{1,2}$, D. Rosińska ${ }^{44,19}$, P. Ruggi ${ }^{15}$, L. Salconi ${ }^{15}$, B. Sassolas ${ }^{30}$, D. Sentenac ${ }^{15}$, V. Sequino ${ }^{10,6}$, Y. Setyawati ${ }^{22,4}$, K. Siellez ${ }^{23}$, M. Sieniawska ${ }^{19}$, A. Singhal ${ }^{5}$, F. Sorrentino ${ }^{21}$, N. Straniero ${ }^{30}$, G. Stratta ${ }^{25,26}$, B. L. Swinkels ${ }^{15}$, M. Tacca $^{12}$, S. Tiwari ${ }^{5,35}$,

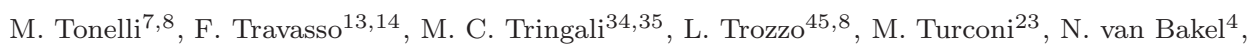
M. van Beuzekom ${ }^{4}$, J. F. J. van den Brand ${ }^{29,4}$, C. Van Den Broeck ${ }^{4}$, L. van der Schaaf ${ }^{4}$, J. V. van Heijningen ${ }^{4}$, M. Vardaro ${ }^{17,18}$, M. Vasúth ${ }^{16}$, G. Vedovato ${ }^{18}$, D. Verkindt ${ }^{3}$,

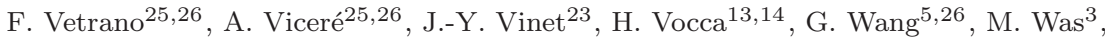


L.-W. Wei ${ }^{23}$, M. Yvert ${ }^{3}$, A. Zadrożny ${ }^{39}$, L. Zangrando ${ }^{18}$, J.-P. Zendri ${ }^{18}$

${ }^{1}$ Università di Salerno, Fisciano, I-84084 Salerno, Italy

${ }^{2}$ INFN, Sezione di Napoli, Complesso Universitario di Monte S.Angelo, I-80126 Napoli, Italy

${ }^{3}$ Laboratoire d'Annecy-le-Vieux de Physique des Particules (LAPP), Université Savoie Mont Blanc, CNRS/IN2P3, F-74941 Annecy-le-Vieux, France

${ }^{4}$ Nikhef, Science Park, 1098 XG Amsterdam, The Netherlands

${ }^{5}$ INFN, Gran Sasso Science Institute, I-67100 L'Aquila, Italy

${ }^{6}$ INFN, Sezione di Roma Tor Vergata, I-00133 Roma, Italy

${ }^{7}$ Università di Pisa, I-56127 Pisa, Italy

${ }^{8}$ INFN, Sezione di Pisa, I-56127 Pisa, Italy

${ }^{9}$ LAL, Univ. Paris-Sud, CNRS/IN2P3, Université Paris-Saclay, Orsay, France

${ }^{10}$ Università di Roma Tor Vergata, I-00133 Roma, Italy

${ }^{11}$ INFN, Sezione di Roma, I-00185 Roma, Italy

${ }^{12}$ APC, AstroParticule et Cosmologie, Université Paris Diderot, CNRS/IN2P3, CEA/Irfu, Observatoire de Paris, Sorbonne Paris Cité, F-75205 Paris Cedex 13, France

${ }^{13}$ Università di Perugia, I-06123 Perugia, Italy

${ }^{14}$ INFN, Sezione di Perugia, I-06123 Perugia, Italy

${ }^{15}$ European Gravitational Observatory (EGO), I-56021 Cascina, Pisa, Italy

${ }^{16}$ Wigner RCP, RMKI, H-1121 Budapest, Konkoly Thege Miklós út 29-33, Hungary

${ }^{17}$ Università di Padova, Dipartimento di Fisica e Astronomia, I-35131 Padova, Italy

${ }^{18}$ INFN, Sezione di Padova, I-35131 Padova, Italy

${ }^{19}$ CAMK-PAN, 00-716 Warsaw, Poland

${ }^{20}$ Università degli Studi di Genova, I-16146 Genova, Italy

${ }^{21}$ INFN, Sezione di Genova, I-16146 Genova, Italy

${ }^{22}$ Department of Astrophysics/IMAPP, Radboud University Nijmegen, P.O. Box 9010, 6500 GL Nijmegen, The Netherlands 
${ }^{23}$ Artemis, Université Côte d'Azur, CNRS, Observatoire Côte d'Azur, CS 34229, Nice cedex 4, France

${ }^{24}$ Institut de Physique de Rennes, CNRS, Université de Rennes 1, F-35042 Rennes, France ${ }^{25}$ Università degli Studi di Urbino 'Carlo Bo', I-61029 Urbino, Italy ${ }^{26}$ INFN, Sezione di Firenze, I-50019 Sesto Fiorentino, Firenze, Italy

${ }^{27}$ Laboratoire Kastler Brossel, UPMC-Sorbonne Universités, CNRS, ENS-PSL Research University, Collège de France, F-75005 Paris, France

${ }^{28}$ Astronomical Observatory Warsaw University, 00-478 Warsaw, Poland

${ }^{29} \mathrm{VU}$ University Amsterdam, $1081 \mathrm{HV}$ Amsterdam, The Netherlands

${ }^{30}$ Laboratoire des Matériaux Avancés (LMA), CNRS/IN2P3, F-69622 Villeurbanne, France

${ }^{31}$ Université Claude Bernard Lyon 1, F-69622 Villeurbanne, France

${ }^{32}$ Università di Napoli 'Federico II', Complesso Universitario di Monte S.Angelo, I-80126 Napoli, Italy

${ }^{33}$ Università di Roma 'La Sapienza', I-00185 Roma, Italy

${ }^{34}$ Università di Trento, Dipartimento di Fisica, I-38123 Povo, Trento, Italy

${ }^{35}$ INFN, Trento Institute for Fundamental Physics and Applications, I-38123 Povo, Trento, Italy

${ }^{36}$ National Astronomical Observatory of Japan, 2-21-1 Osawa, Mitaka, Tokyo 181-8588, Japan

${ }^{37}$ INAF, Osservatorio Astronomico di Capodimonte, I-80131, Napoli, Italy

${ }^{38}$ University of Biatystok, 15-424 Biatystok, Poland

${ }^{39}$ NCBJ, 05-400 Świerk-Otwock, Poland

${ }^{40}$ IM-PAN, 00-956 Warsaw, Poland

${ }^{41}$ ESPCI, CNRS, F-75005 Paris, France

${ }^{42}$ Università di Camerino, Dipartimento di Fisica, I-62032 Camerino, Italy

${ }^{43}$ Université de Lyon, F-69361 Lyon, France

${ }^{44}$ Janusz Gil Institute of Astronomy, University of Zielona Góra, 65-265 Zielona Góra, Poland

${ }^{45}$ Università di Siena, I-53100 Siena, Italy

*e-mail: viviana.fafone@roma2.infn.it 
The Advanced Virgo project was funded in 2009 with the aim of improving the sensitivity of the Virgo interferometric detector for gravitational waves by a factor of ten, which corresponds to an increase in the detection rate by about three orders of magnitude. The upgrade is now close to completion: the new interferometer will enter its commissioning phase in 2016. The new detector will be hosted in the same infrastructure as Virgo, but many technological upgrades have been put in place to reach the sensitivity goal. In this paper the detector design and the observational perspectives are discussed.

Keywords: Gravitational waves; detectors.

\section{Introduction}

Advanced Virgo (AdV) is a second generation interferometer (ITF) for the search of Gravitational Waves (GW). Its design sensitivity will allow to detect astrophysical sources of gravitational radiation such as binary neutron stars (1.4 solar masses each) up to $140 \mathrm{Mpc}$ or 30 solar masses coalescing binary black holes as far as 1 Gpc. AdV will be part of the international network of GW interferometers: in 2014 a Memorandum of Understanding for full data exchange, joint data analysis and publication policy with the LIGO Scientific Collaboration was renewed, thus strengthening the world-wide network of second generation detectors.

\section{Detector overview}

The hosting infrastructure for AdV is the same as for Virgo, ${ }^{1}$ but relevant modifications have been implemented to make it compliant with the requirements of $\mathrm{AdV}$. In particular, large cryotraps at the ends of the $3 \mathrm{~km}$ pipes have been installed, in order to lower the residual pressure by a factor of about 100, and acoustically isolated clean rooms have been built to host the laser, the input optics and the detection system. Many other important changes in the detector have been introduced in order to improve its sensitivity by a factor of ten. In the following, the main features of Advanced Virgo are presented. A more exhaustive description can be found in the AdV Technical Design Report ${ }^{2}$ and in a recent review. ${ }^{3}$

\subsection{Interferometer configuration}

The optical configuration of $\mathrm{AdV}$ has been defined to get the best possible sensitivity, compatibly with the Virgo infrastructure: Fabry-Perot (FP) arm cavities $3 \mathrm{~km}$ long and recycling cavities $\sim 12 \mathrm{~m}$ long. AdV will be a dual-recycled ITF where, besides the power recycling (PR) cavity, a signal-recycling (SR) cavity will be present (see Fig. 1). The tuning of the SR parameter allows to change the shape of the sensitivity curve and optimise it for different astrophysical sources.

The design of the arm cavities aims to reduce the mirror coating thermal noise which limits the detector sensitivity in the mid-frequency range. To this purpose, the beam size on the input test masses has been increased from $2 \mathrm{~cm}$ to $5 \mathrm{~cm}$, corresponding to clipping losses less than $1 \mathrm{ppm}$ on the $350 \mathrm{~mm}$ mirror diameter. 


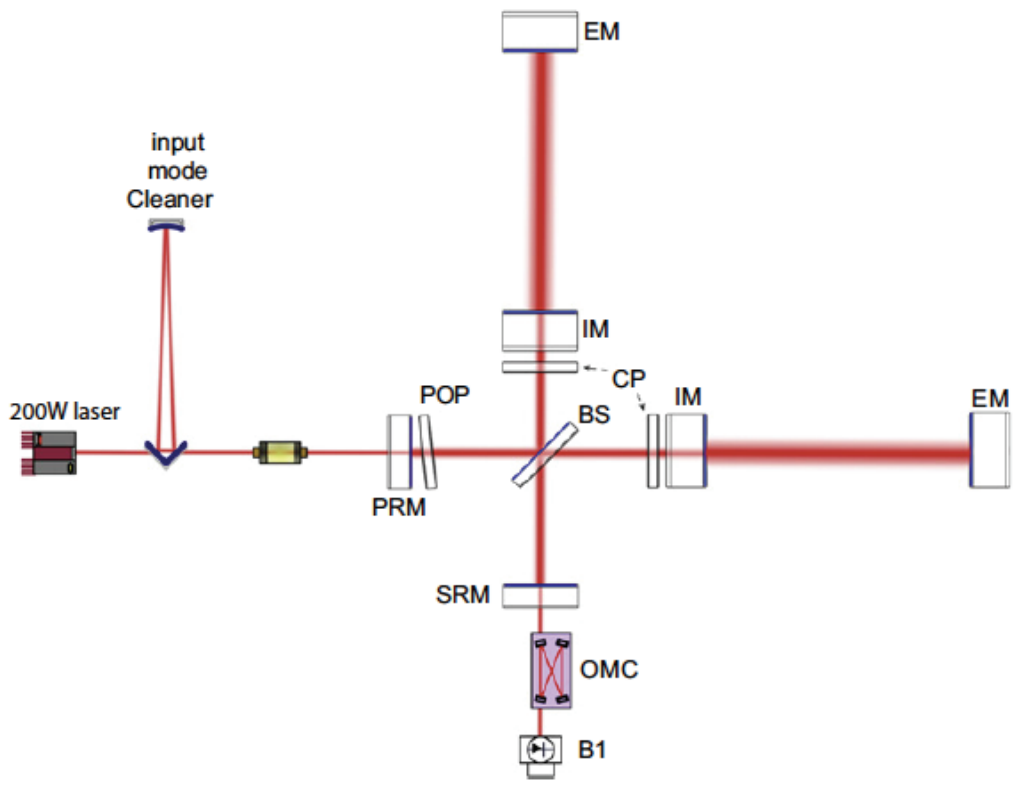

Fig. 1. A schematic view of the Advanced Virgo optical layout.

The arm cavities mirrors have a concave RoC slightly larger than half the armcavity length to ensure cavity stability and the beam waist is placed almost half way between the input and end mirrors.

The recycling cavities are marginally stable. In this configuration, conceptually similar to the one used in Virgo, the Gouy phase accumulated during the free-space propagation inside the cavity is not sufficient to separate the high-order modes from the fundamental one. Moreover, the increased beam size further reduces the round-trip Gouy phase and hence increases the degeneracy. The degeneracy makes the recycling cavity extremely sensitive to optical aberrations, arising from optics defects and from heating of the optics themselves, due to absorption of a tiny (order of ppm) fraction of the stored power. These aberrations detrimentally affect interferometer performance since they, by definition, represent a change in the interferometer optical system from the ideal design. To reduce the impact of these effects on the detector performances, an adaptive correction system (named Thermal Compensation System) has been implemented. ${ }^{4}$ The system includes Hartmann sensors and phase cameras to measure the aberrations, ring heaters $(\mathrm{RH})$ around several suspended optics to change the radius of curvature, and CO2 laser projectors, which shine on dedicated compensation plates $(\mathrm{CP})$, to correct thermal lensing and optical defects. 


\subsection{High power laser, input and output optics}

The Advanced Virgo design comprises for its final configuration a $200 \mathrm{~W}$ continuouswave laser, for the reduction of the shot noise in the high frequency range, resulting in $125 \mathrm{~W}$ at the interferometer input. The corresponding optical power stored in the Fabry-Perot cavities, for a finesse of 450, will be around $700 \mathrm{~kW}$, a factor of about 35 higher with respect to Virgo. The laser has to be stabilized in frequency, intensity and beam pointing, in order to bring its technical noises down to a level where they no longer spoil the detector sensitivity. The laser frequency fluctuations cannot exceed a few $\mu \mathrm{Hz} / \sqrt{\mathrm{Hz}}$, the power fluctuations must be in the range of $10^{-9} / \sqrt{\mathrm{Hz}}$ and the beam pointing has to be below $10^{-11} \mathrm{rad} / \sqrt{\mathrm{Hz}}$. The $200 \mathrm{~W}$ laser, based on fiber amplifiers, will be installed after 2018, while during the first years of operation a medium-power laser will be used: an Nd:YVO4 (neodymiumdoped yttrium orthovanadate) oscillator amplifying a $20 \mathrm{~W}$ injection-locked laser to deliver around $60 \mathrm{~W}$.

The laser light is injected into the interferometer through a system of optics that have to provide a beam with the required power, geometrical shape, frequency and angular stability. The input optics for $\mathrm{AdV}$ must be compliant with the increased optical power. To this purpose, custom electro-optic modulators and Faraday isolators have been developed and a DC readout scheme will be used, requiring a new design of the output mode cleaner of the detection system. Another upgrade of the detection system concerned the suspension under vacuum of the output mode matching telescope and of the photodetectors to be used in science mode, in order to minimise the impact of scattered-light noise introduced into the ITF through the displacement of the optics.

\subsection{Mirrors}

The AdV input and end mirrors have the same diameter as the Virgo mirrors $(35 \mathrm{~cm})$ but are twice as thick $(20 \mathrm{~cm})$ and twice as heavy $(42 \mathrm{~kg})$. The beam splitter is $55 \mathrm{~cm}$ in diameter and $6.5 \mathrm{~cm}$ thick. Power/signal recycling mirrors $(\mathrm{PRM} / \mathrm{SRM})$ are $35 \mathrm{~cm}$ in diameter and $10 \mathrm{~cm}$ thick. A new material for the input mirrors and the beam splitter substrates (Suprasil 3002/3001) has been used, with very low optical absorptions $\left(0.2 \mathrm{ppm} \mathrm{cm}^{-1}\right.$ at $\left.1064 \mathrm{~nm}\right)$, in order to reduce the thermal effects. The substrates of the other mirrors, which are less sensitive to thermal effects, are made of Suprasil 312, a fused silica grade of lower optical quality (and cost), with measured absorptions lower than $1 \mathrm{ppm} \mathrm{cm}^{-1}$. The polishing of the mirrors has been done in order to guarantee the needed flatness and microroughness. Both effects contribute to the scattering of light from the fundamental mode to higher order modes and generate losses and extra noise. To meet the round-trip losses requirement in the $\mathrm{AdV}$ cavities (75 ppm) a flatness requirement of $0.5 \mathrm{~nm} \mathrm{rms}$ on $150 \mathrm{~mm}$ diameter for the arm-cavity mirrors (IM, EM) was set. The flatness specifications for all of the other optics were set to be lower than $2 \mathrm{~nm}$ rms on a $150 \mathrm{~mm}$ diameter. 
The mirror coating makes use of $\mathrm{Ta}_{2} \mathrm{O}_{5}$ doped with $\mathrm{Ti}$ as the high refractive index material that, together with the optimised layers thickness, improves the mirrors mechanical losses. The coating must not spoil the flatness requirements set for the polishing. In order to get the required uniformity on two large substrates at the same time a planetary motion coupled to a masking technique has been developed and applied to the AdV mirrors. Since an auxiliary green laser will be used to lock the interferometer, the mirror coatings have been designed to have the optical properties suitable for this purpose.

\subsection{Mirror suspension and isolation}

All the core optics of AdV are integrated in a complex isolation system, the socalled Superattenuator (SA), a hybrid (passive-active) attenuation system, capable of reducing seismic noise by more than 10 orders of magnitude in all six degrees of freedom above a few Hz. The SA already implemented in Virgo was compliant with the AdV requirements. For this reason, no conceptual changes in the mechanical design have been introduced. The most relevant interventions concerned some upgrades to increase the SA reliability in conditions of high seismicity, such as the possibility to control the ground tilt, and a new design of the last seismic filter of the SA chain, to make it compliant with the last stage comprising the mirror and its position control system, known as the payload. This has been in fact redesigned to take into account the necessity to host heavier mirrors and additional components: the $\mathrm{CP}$ and the $\mathrm{RH}$ of the thermal compensation system, and the baffles for stray light mitigation that could be in fact a significant limitation on detector sensitivity.

\section{Present status and further evolution}

At the time of writing (March 2016), the installation of the upgraded components is in its final phase. Commissioning of some sub-systems already started and will progressively evolve toward the commissioning of the full interferometer. The goal of the AdV commissioning is to join the second observational run of LIGO (O2), foreseen at the end of 2016.

$\mathrm{AdV}$ will not initially be operated in the final configuration. The increased complexity of the detector will in fact require a step-by-step approach, corresponding to steps of increasing complexity. Periods of commissioning will be alternated with periods of data-taking and such plans will be coordinated with Advanced LIGO in order to maximise the capabilities of the network.

Some reference steps have been identified, with a corresponding observing roadmap. ${ }^{5}$ The figure of merit considered to describe the detector sensitivity is the binary neutron star (BNS) range, defined as the volume-and-orientation-averaged distance at which a compact binary coalescence consisting of two 1.4 solar masses neutron stars gives a matched filter signal-to-noise ratio (SNR) of 8 in a single detector. ${ }^{6}$ The sensitivity curves for the various AdV configurations are shown in Fig. 2. 


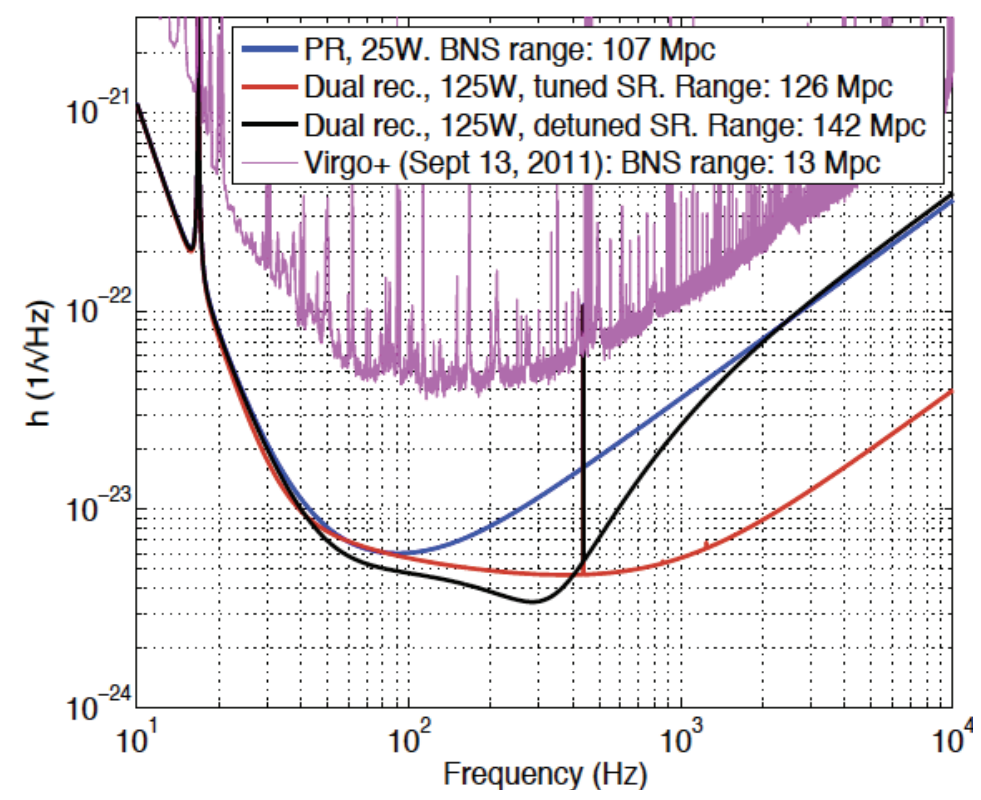

Fig. 2. Planned evolution of the Advanced Virgo sensitivity over the next decade.

The two Advanced LIGO detectors had a first four months data taking run (the O1 run) which ended on January 12, 2016. The detectors were not at full design sensitivity, with both instruments running with a 60-80 Mpc BNS range. During this run the first detection of GWs occurred. ${ }^{7}$ The evolution of the observations will see increasing duration and sensitivity runs.

A plausible scenario for the operation of the LIGO-Virgo network over the next decade is the following: ${ }^{5}$

- 2016-2017: (O2) A six-month run with the LIGO detectors at 80-120 Mpc and $\mathrm{AdV}$ at 20-60 Mpc.

- 2017-2018: (O3) A nine-month run with the LIGO detectors at 120-170 $\mathrm{Mpc}$ and AdV at 60-85 Mpc.

- 2019+: Three-detector network with LIGO at full sensitivity of $200 \mathrm{Mpc}$ and Virgo at 65-115 Mpc.

- 2022 +: Full network of the two LIGO detectors and AdV at design sensitivity (aLIGO at $200 \mathrm{Mpc}, \mathrm{AdV}$ at $130 \mathrm{Mpc}$ ), with other detectors potentially joining the network. Including a fourth detector improves sky localization. ${ }^{8}$ More detectors are indeed expected to join the international network: Kagra in $\operatorname{Japan}^{9}$ is being built and LIGO India ${ }^{10}$ has been recently approved. 


\section{Conclusion}

The upgrade of Virgo to its Advanced configuration is in the final stage. The interferometer is now starting the commissioning that will allow to enter data taking in 2016, joining Advanced LIGO in the second observational run (O2). The sensitivity of the network of interferometric detectors will evolve over the next decade, opening new perspectives and making GW astronomy an exciting and fruitful adventure.

\section{References}

1. The Virgo Collaboration, JINST 7, 3012 (2012).

2. The Virgo Collaboration, Advanced Virgo technical design report Virgo Internal Document VIR-0128A-12 (2012) https://tds.ego-gw.it/itf/tds/file.php?callFile=VIR-0128A-12.pdf

3. The Virgo Collaboration, Class. Quantum Grav. 32, 024001 (2015).

4. A. Rocchi, E. Coccia, V. Fafone, V. Malvezzi, Y. Minenkov, L. Sperandio, Journal of Physics: Conference Series 363, 012016 (2012).

5. The LIGO Scientific Collaboration and the Virgo Collaboration, Living Rev. Relativity 19, (2016).

6. L.S. Finn and D.F. Chernoff, Phys. Rev. D 47, 2198-2219 (1993).

7. The LIGO Scientific Collaboration and the Virgo Collaboration, $P R L$ 116, 061102 (2016).

8. S. Klimenko et al., Phys. Rev. D 83, 102001 (2011).

9. Y. Aso, Y. Michimura, K. Somiya, M. Ando, O. Miyakawa, T. Sekiguchi, D. Tatsumi and H. Yamamoto, (KAGRA Collaboration), Phys. Rev. D 88, 043007 (2013).

10. B.R. Iyer, T. Souradeep, C.S. Unnikrishnan, S. Dhurandhar, S. Raja, and A. Sengupta, (IndIGO Consortium), LIGO-India, M1100296-v2, (IndIGO, India, 2011). 